\title{
Nivolumab plus ipilimumab combination therapy for the first-line treatment NSCLC: evidence to date
}

This article was published in the following Dove Press journal: Cancer Management and Research

\author{
Jordi Remon' \\ Laura Esteller' \\ Álvaro Taus ${ }^{2,3}$ \\ 'Centro Integral Oncología Clara Campal \\ Barcelona, HM-Delfos, Medical Oncology \\ Department, Barcelona, Spain; ${ }^{2}$ Hospital \\ del Mar, Medical Oncology Department, \\ Barcelona, Spain; ${ }^{3}$ Oncology Department, \\ Cancer Research Program, IMIM \\ (Hospital del Mar Medical Research \\ Institute), Barcelona, Spain
}

\begin{abstract}
Immune checkpoint inhibitors (ICI) as monotherapy in selected patients as well as in combination with chemotherapy have become the standard of care in the first-line treatment strategy of advanced non-small cell lung cancer (NSCLC) patients. Combination treatment with ICI, such as nivolumab and ipilimumab or durvaluamb and ipilimumab, has also been proposed as potential strategies in this setting in selected advanced NSCLC patients. Characterizing predictive markers of long-term clinical benefit with ICI is a critical objective. Tumor mutational burden has been proposed as a potential predictive biomarker. In this review, we discuss the efficacy of nivolumab and ipilimumab in advanced NSCLC patients as well as the clinical utility of tumor mutational burden in the efficacy of this combination. Ongoing clinical trials with nivolumab and ipilimumab, and the efficacy of this combination in subgroups of NSCLC patients, such as elderly patients and patients with brain metastases, are also discussed.
\end{abstract}

Keywords: nivolumab, ipilimumab, tumor mutational burden, CheckMate trial, non-small cell lung cancer

\section{Introduction}

In the last decade, several immune checkpoint inhibitors (ICIs) have become the standard of care approved by the US Food and Drug Administration (FDA) and European Medicines Agency (EMA) in the treatment of advanced non-small cell lung cancer (NSCLC), either in the first-line setting (as monotherapy in selected patients ${ }^{1}$ or in combination with chemotherapy regardless the histologic subtype ${ }^{2-4}$ ) or beyond, mainly as monotherapy treatment. ${ }^{5-8}$ Other strategies, such as the combination of ICIs, have also been assessed in selected patients, ${ }^{9}$ but this strategy has not already been approved by health authorities as the standard of care. ICIs have shifted the prognosis of advanced NSCLC patients, therefore, characterizing predictive markers of long-term clinical benefit with ICI is a critical objective, mainly for avoiding potential toxicity and to limit economic expenses. There are plenty of data confirming the predictive, albeit imperfect, ability of programmed death-ligand 1 (PD-L1) expression to identify NSCLC patients with most favorable outcomes with ICI. ${ }^{10-12}$ Despite its controversial results and constraints based on the responses observed in PD-L1 negative tumors, ${ }^{2,4,6,8}$ PD-L1 expression measured by immunohistochemistry is currently the main scaffold decision-making tool used in clinical practice for selecting those patients deriving the most benefit from ICIs at least in a first-line setting. ${ }^{1}$ Tumor mutational burden (TMB) has recently
Correspondence: Jordi Remon Centro Integral Oncológico Clara Campa Barcelona, HM Delfos, Medical Oncology Department, Avinguda de Vallcarca, I5I,

Barcelona 08023, Spain

Tel +34932545000

Email jordi.remon@delfos.cat 
emerged as a biomarker, independent of PD-L1 expression, to identify patients who derive clinical benefit from anti-programmed death-1 (anti-PD-1) monotherapy, nivolumab, or the combination of anti-PD(L)1 and cytotoxic T-lymphocyte-associated antigen (CTLA-4) antibody. ${ }^{9,13-15}$ In this comprehensive review, we discuss the efficacy of nivolumab and ipilimumab in advanced NSCLC patients, as well as the clinical utility of TMB in the efficacy of this combination. Ongoing clinical trials with nivolumab and ipilimumab, and the efficacy of this combination in subgroups of NSCLC patients, such as elderly patients and patients with brain metastases, are also discussed.

\section{Tumor mutational burden and other biomarkers}

Cancer is a genetic disease as a consequence of the accumulation of somatic mutations into the DNA of the affected cells. These mutations may generate neoantigens (tumor-specific, mutated peptides presented in the surface of cancer cells) that should be adequately processed and presented by the major histocompatibility complex (MHC), and afterward these neoantigens should be recognized by T-cells as non-self to achieve adequate antitumor T-cell response, ${ }^{16}$ stimulating the patient's immune system to reject the tumor. However, not all mutations will generate neoantigens. In fact, only a minority of mutations generate peptides that are properly processed and loaded on to MHC complexes, and even fewer are able to be recognized by $\mathrm{T}$ cells. ${ }^{17}$ The amount of neoantigens correlates with the probability to enhance the T-cell response. Although only a minority of these mutations will generate neoantigens, ${ }^{18}$ the hypothesis is that the chances of a neoantigen existing will be increased if more nonsynonymous mutations are present in the tumor. Therefore, tumor mutational burden (TMB), defined as the number of non-synonymous alterations (somatic, coding, base substitutions, and short indels) per megabase (mut/MB) of genome examined, can represent a useful estimation of tumor neoantigen load. ${ }^{17}$

TMB can be determined through whole exome sequencing (WES) or targeted next-generation sequencing (NGS) and it is defined as either the total number of somatic mutations (Mut) in the tumor exome (WES) or the total number of synonymous and non-synonymous mutations per megabase (Mb) present at $\geq 5 \%$ allele frequency in the sequenced tumor genome by NGS (FoundationOne CDx assay). Results are expressed as Mut/Mb. Major limitations for using WES in daily clinical practice include its cost, and the facts that this technique is time consuming, labor intensive, and needs large sequencing capacity; for these reasons, NGS is the most commonly used technique for extrapolating the TMB in daily clinical practice, usually with a limited number of genes. ${ }^{16}$ Although TMB quantified by targeted NGS correlates with that of WES, ${ }^{19,20}$ caution may be needed when using smaller panels. TMB is a continuous variable, so different assays and different definitions of high TMB will alter the population size and then the extent of treatment benefit in the identified biomarker-positive group. $^{21}$ Therefore, the standardization of TMB calculation and reporting, as well as a universal threshold for defining high TMB, are current challenges. ${ }^{16}$ Of note, not all neoantigens may cause an immune response. Neoantigens may be a result of previous cytotoxic therapy, which can increase the abundance of branch mutations and consequently TMB but cannot trigger an immune response. ${ }^{22}$

TMB has been associated with improved survival in patients receiving ICI across a wide variety of cancer types. $^{23}$ The variance of TMB number is high between different tumor types, and NSCLC is usually associated with high TMB. ${ }^{24,25}$ TMB is particularly increased in smokers and is noteworthy in metastases as compared to its primary counterparts. ${ }^{26,27}$ Importantly, TMB and PDL1 expression appear to be independent predictors of response to ICIs. ${ }^{19,26}$ However, it has been reported that the use of combined biomarkers, PD-L1 and TMB, may result in predictive synergism. ${ }^{13}$ Different clinical trials have assessed the predictive value of TMB in different nivolumab plus ipilimumab combination trials, which will be detailed in the next section of this manuscript.

As an alternative to tissue, TMB has been also assessed in circulating tumoral DNA (ctDNA) from blood/plasma (bTMB). In one retrospective study in NSCLC, bTMB was determined using a 394-gene panel and was compared to tissue TMB (FoundationOne CDx assay) and to the FoundationACT (FACT) dedicated to ctDNA assay (including only 62 genes). Out of 259 patients were evaluable for both bTMB and tissue TMB. Overall agreement and positive percent agreement (PPA) were $81.5 \%$ and $63.6 \%$, respectively, when using the 394-gene panel for bTMB. However, when the FACT assay was compared to tissue TMB, PPA dropped to $17 \%$, suggesting a sufficiently sized panel is required to sensitively identify patients with high TMB. However, the performance on variant detection was similar when overlapping allele regions were compared: $93 \%$ of variants were detected in 
both assays. $^{28}$ The predictive value of bTMB in NSCLC patients for the efficacy of ICI as monotherapy has been retrospectively assessed in second-line ${ }^{29}$ and prospectively in the first-line setting. ${ }^{30}$ Similarly, the predictive value of bTMB in trials assessing the combination of ICI, including nivolumab and ipilimumab, is discussed in the next section of this manuscript. It is noteworthy that improved understanding of pharmacodynamic effects of these agents in patients will support rational development of immunebased combinations against cancer ${ }^{31}$ and understand the multiple cellular mechanisms underlying the synergistic benefit of this combination may help to enhance the efficacy of this strategy. ${ }^{32}$

Beyond PD-L1 and TMB, there may be other important potential predictive biomarkers. MSI, a pattern of hypermutation that occurs due to defects in the mismatch repair system, has also been identified as an independent predictor of response to immunotherapy regardless of the cancer'stissue of origin. ${ }^{33}$ However, its rarity (only a small fraction of $3.8 \%$ of cancers and $1 \%$ of NSCLC), ${ }^{34}$ impairs its use, at least in the NSCLC population, as a unique clinical ICI biomarker. The presence of mutations in genes such as JAK1, JAK 2, B2M, STK11, SERPINB3 and SERPINB4, as well as some immune evasion mechanisms like transforming growth factor beta signaling or indoleamine 2,3-dioxygenase (IDO) activity, may influence response to ICIs. ${ }^{35-38}$ The role of all these potential biomarkers must be validated in prospective trials. The T-cell-inflamed gene expression profile, ${ }^{39}$ immune gene expression signatures, ${ }^{40,41}$ as well as a description of the microbiome ${ }^{42-44}$ also represent emerging predictive biomarkers that have to be validated prospectively. In this setting, the CheckMate 592 (NCT03001882) is a two-part, exploratory, open-label phase II study exploring potential biomarkers, including PD-L1 and TMB, and their association with clinical benefit with first-line nivolumab and ipilimumab for stage IV or recurrent NSCLC. In part 1, approximately 100 patients will be analyzed at baseline according to PD-L1 status ( $\geq 1 \%$ vs $<1 \%$ ). In part 2, approximately 150 patients will be treated regardless of PD-L1 status. The primary endpoint in part 1 is the association of response rate (RR) with baseline TMB, along with candidate peripheral blood and tumor biomarkers at baseline and on treatment; as well as outcome according toprogression-free survival(PFS) and overall survival (OS). Primary endpoints in part 2 are the association of RR with baseline tissue and blood TMB, and secondary endpoints are RR, PFS, OS, safety, and the association of enteric biomarkers with efficacy. ${ }^{45}$ This trial will help to elucidate other potential predictive biomarkers with clinical utility in daily clinical practice for patient selection.

\section{Clinical trials with nivolumab and ipilimumab Phase I clinical trials}

CheckMate 012 (NCT01454102) is a multicohort phase I study that was designed to evaluate nivolumab as monotherapy or in combination with other agents, including ipilimumab, as first-line treatment for advanced NSCLC patients. $^{46-49}$ In the cohort assessing the safety and efficacy of the combination of nivolumab and ipilimumab, different schedules were evaluated in different amendments of the protocol. Finally, according to tolerability and safety concerns, patients were randomly assigned (1:1:1) to receive nivolumab $1 \mathrm{mg} / \mathrm{kg}$ every 2 weeks plus ipilimumab $1 \mathrm{mg}$ / $\mathrm{kg}$ every 6 weeks, or nivolumab $3 \mathrm{mg} / \mathrm{kg}$ every 2 weeks plus ipilimumab $1 \mathrm{mg} / \mathrm{kg}$ either every 12 weeks or every 6 weeks until disease progression, unacceptable toxicities, or withdrawal of consent. Data from the two nivolumab at $3 \mathrm{mg} / \mathrm{kg}$ every 2 weeks cohorts were considered potentially suitable for further clinical development, and we will focus on published results on efficacy and safety for this combination. $^{46}$

A total of 78 patients were randomized to receive nivolumab plus ipilimumab every 12 weeks (Q12W, N=38) or every 6 weeks (Q6W, N=39). Patients with adequately treated and asymptomatic brain metastases (BM) were eligible as well as patients harboring EGFR mutations (10\% of enrolled patients). Patients were not selected according to PD-L1 expression or other biomarkers. However, PD-L1 expression (by clone 28-8 IHC) was assessed retrospectively in fresh or archival pretreatment tumor samples. ${ }^{46}$ Baseline PD-L1 expression was quantifiable in $90 \%$ of patients $(\mathrm{N}=66)$ in $\mathrm{Q} 12 \mathrm{~W} / \mathrm{Q} 6 \mathrm{~W}$ cohorts; of these, 47 $(68 \%)$ and $13(19 \%)$ patients had $\geq 1 \%$ and $\geq 50 \%$ PD-L1 expression, respectively. ${ }^{50}$ Similar proportions of grade 3-4 treatment-related adverse events (AEs) were reported in both cohorts (37\% in the Q12W vs 33\% in Q6W); with the most commonly reported grade $\geq 3$ AEs increasing lipase, pneumonitis, adrenal insufficiency and colitis. Treatment-related serious AEs were reported in $32 \%$ and 28\% of Q12W and Q6W arms, respectively, with a similar proportion of patients in both arms who discontinued treatment as a consequence of treatment-related AEs (11\% and $13 \%$, respectively). No treatment-related deaths occurred. ${ }^{46}$ Efficacy was similar in both schedule arms with 
a confirmed response rate (RR) of $47 \%$ in the Q12W arm and 38\% in the Q6W arm. It is noteworthy that progressive disease was reported in $13 \%$ and $28 \%$ of patients of both arms, respectively. However, disease progression in Q6W arm occurred earlier, with $44 \%$ of patients experiencing progression or dying before the first imaging assessment, compared with $18 \%$ in Q12W arm. Globally, these results suggest a real risk of hyper-progressive disease on treatment with the combination rather than suggesting intrinsic differences in clinical activity between ipilimumab given Q6W or $\mathrm{Q} 12 \mathrm{~W}$. The median duration of response was not reached in either cohort. Median PFS was longer in the Q12W arm compared with Q6W arm (8.1 months vs 3.9 months). ${ }^{46}$ The magnitude of clinical benefit achieved with the combination treatment was enhanced with higher PD-L1 expression. Pooling the two cohorts and after 2 years of follow-up, the RR was $43 \%$, reaching $57 \%$ and $92 \%$ in patients with $\geq 1 \%(\mathrm{~N}=47)$ and $\geq 50 \%$ PD-L1 expression ( $\mathrm{N}=13)$, respectively. Similarly, the PFS was longer among tumors with PD-L1 expression, with a 2-year PFS of $29 \%$ in the whole population, reaching $38 \%$ and $54 \%$ in tumors with PD-L1 expression $\geq 1 \%$ and $\geq 50 \%$, respectively. Finally, the 2 -year OS was also enhanced in PD-L1 positive tumors, being of $49 \%$ in the whole population, and increasing to $58 \%$ and $62 \%$ for PD-L $1 \geq 1 \%$ and PD-L $1 \geq 50 \%$ patients. ${ }^{50}$ The study was not powered to directly compare safety and efficacy between both treatment schedules due to a limited number of patients and imbalances in baseline relevant clinical characteristics because of the lack of stratification. ${ }^{50}$ In the pooled cohort, $44 \%$ of patients achieved 2-year survival or longer. These patients compared with the whole population trend toward being more current/former smokers and PDL1 positive. ${ }^{50}$ TMB by WES was assessed in 75 patients enrolled in the CheckMate 012 trial, demonstrating the association between TMB high ( $>$ median, 158 mutations) vs low ( $<$ median) and the efficacy of nivolumab an ipilimumab in terms of RR ( $51 \%$ vs $31 \%, p=0.0005)$ and PFS
(HR 0.41, 95\%ci: 0.23-0.73, $p=0.0024)^{51}$ (Table 1). Globally, these results suggested a better outcome was attained with the combination of nivolumab and ipilimumab in tumors with PD-L1 expression and high TMB. ${ }^{50,51}$ The CheckMate 012 trial endorsed a potential clinical activity synergism and tolerable safety profile with the combination, supporting further assessment of this combination in a phase III study. After integrating observations from other tumor types in which greater ipilimumab exposure was associated with improved activity, the nivolumab $3 \mathrm{mg} /$ $\mathrm{kg}$ Q2W plus ipilimumab $1 \mathrm{mg} / \mathrm{kg}$ Q6W regimen was chosen for further development in NSCLC. ${ }^{46}$

\section{Phase II trials}

In the single-arm, phase II CheckMate 568 trial, 288 chemotherapy-naive stage IV NSCLC patients received nivolumab $3 \mathrm{mg} / \mathrm{kg}$ Q2W plus ipilimumab $1 \mathrm{mg} / \mathrm{kg}$ Q6W for up to 2 years. EGFR- and $A L K$-targetable NSCLC patients were not allowed. The primary endpoint was objective response rate (ORR) in patients with $1 \%$ or more and less than 1\% tumor PD-L1 expression. Efficacy according to the TMB assessed by FoundationOne CDx assay was a secondary endpoint. TMB classification performance with receiver operating characteristic (ROC) curves was used to determine an appropriate and clinically validated TMB cut-off associated with enhanced activity of immunotherapy combination in the first-line setting in NSCLC patients. ${ }^{14}$ Of treated patients with tumor available for testing, 252 patients $(88 \%)$ of 288 were evaluable for PD-L1 expression (55\% PD-L1 $\geq 1 \%$ and $45 \%$ PD-L1 $<1 \%$ ) and 98 patients (34\%) for TMB ( $49 \%$ with $\mathrm{TMB} \geq 10$ mutations per megabase, Mut $/ \mathrm{Mb}$ ). In all treated patients, the RR was $30 \%$, reaching $41 \%$ in patients with $1 \%$ or greater and $15 \%$ in patients with less than $1 \%$ tumor PD-L1 expression. Median PFS was longer (6.8 months vs 2.8 months) in patients with $1 \%$ or greater vs less than $1 \%$ tumor PD-L1 expression. In the TMB-evaluable population, RR increased

Table I Treatment efficacy with nivolumab plus ipilimumab as first-line treatment in advanced NSCLC patients

\begin{tabular}{|l|l|l|l|l|l|l|}
\hline Trial & Treatment dose & N & RR (\%) & PFS (mo.) & OS (mo.) & $\begin{array}{l}\text { Grade } \geq 3 \text { AE } \\
\text { (\%) }\end{array}$ \\
\hline CheckMate 012 & & & & & \\
& Niv,50 3 Q2W + Ipi I QI2W & 38 & 47 & 8.1 & 2 -year OS: $56 \%$ & 37 \\
& Nivo 3 Q2W + Ipi I Q6W & 40 & 38 & 3.9 & 2 -year OS: $42 \%$ & 33 \\
CheckMate 568 & Nivo 3 Q2W + Ipi I Q6W & 288 & 30 & Note reported & Not reported & 29 \\
CheckMate 227 & Nivo 3 Q2W + Ipi I Q6W & 139 & 45.3 & 7.2 & 23.3 & 31 \\
CheckMate 817 & Nivo 240 mg Q2W+lpi I Q6W & 391 & 35 & 6.1 & Not reported & 35 \\
\hline
\end{tabular}

Notes: Doses of Nivolumab (Nivo.) and Ipilimumab (lpi.) are mg/kg. Q2W/Q6W/Q12W: Every 2/6//2 weeks. *Cohort A

Abbreviations: AE, OS, overall survival; PFS, progression-free survival; RR, response rate. 
in subgroups of patients with higher TMB, plateauing at 10 or more Mut/Mb (RR: $9 \%, 15 \%, 44 \%$, and $39 \%$ in patients with $\mathrm{TMB}<5,5-10, \geq 10$, and $\geq 15 \mathrm{Mut} / \mathrm{Mb}$, respectively). The association of efficacy with TMB did not depend on tumor PD-L1 expression. Regardless of PD-L1 expression, RR was greater in patients with TMB of 10 or more Mut/ $\mathrm{Mb}(42 \% \mathrm{PD}-\mathrm{L} 1 \geq 1 \%$ and $47 \%$ PD-L $1<1 \%$ ) versus TMB of fewer than $10 \mathrm{Mut} / \mathrm{Mb}$ (18\% PD-L1 $\geq 1 \%$ and 5\% PDL $1<1 \%$ ). Median PFS was longer in patients with TMB $\geq 10 \mathrm{Mut} / \mathrm{Mb}, 7.1$ months compared with 2.8 in patients with $\mathrm{TMB}<10 \mathrm{Mut} / \mathrm{Mb}$. ROC analysis of TMB vs RR for nivolumab and ipilimumab demonstrated optimal classification performance at $10 \mathrm{Mut} / \mathrm{Mb}{ }^{14}$ The safety profile for nivolumab and ipilimumab was consistent with previous reports, and no new safety signals were observed, with grade $3 \geq$ AEs in $29 \%$ of patients in the whole population, leading to treatment discontinuation in $9 \%$ of cases. ${ }^{14}$ This cutoff point of TMB was subsequently validated through a preplanned analysis in the randomized phase III CheckMate 227 trial. $^{9}$

\section{Phase III \& IV trials}

Several phase III and IV studies have assessed the activity and safety of dual checkpoint inhibitor blockade in firstline setting of advanced NSCLC patients, such as the CheckMate 227 trial (NCT02477826), CheckMate 817 trial (NCT 02869789), DICIPLE trial (NCT03469960), CheckMate 9LA trial (NCT03215706), the MYSTIC trial (NCT02453282), NEPTUNE trial (NCT 02542293), and POSEIDON trial (NCT03164616).

The phase III CheckMate 227 trial assessed multiple hypotheses regarding the efficacy of nivolumab or nivolumab-based regimens in first-line treatment in biomarkerselected advanced NSCLC patients without EGFR- or $A L K$-positive tumors. The trial randomized 1,739 patients not selected according to PD-L1 expression; however, $68 \%$ of randomized patients $(\mathrm{N}=1189)$ had tumors with PD-L1 expression $\geq 1 \%$ by $28-8$ Dako IHC. ${ }^{9}$ According to emerging data reported in the CheckMate 568 trial regarding the correlation between high TMB and efficacy, ${ }^{14}$ the CheckMate 227 trial protocol was amended and PFS (assessed by blinded independent central review) with nivolumab (3 $\mathrm{mg} / \mathrm{kg} \mathrm{Q} 2 \mathrm{~W}$ ) and ipilimumab (1 $\mathrm{mg} / \mathrm{kg}$ Q6W until PD or toxicity) versus chemotherapy based on tumor histologic type in patients with $\mathrm{TMB} \geq 10 \mathrm{Mut} / \mathrm{Mb}$ (by FoundationOne $\mathrm{CDx}$ assay) regardless of PD-L1 expression was added as coprimary endpoint. The other co-primary endpoint was OS with nivolumab plus ipilimumab in a population selected on the basis of PD-L1 expression. ${ }^{9}$

Of the 1,739 patients enrolled in the trial, only 1,004 (58\%) patients had valid results for assessing TMB. Of them, 44\% were classified as TMB-high (24\% of the intent-to-treat population), and just 299 were selected for evaluating the coprimary PFS endpoint. ${ }^{9}$ Crossover between treatment groups within the trial was not allowed, but almost $30 \%$ of patients assigned to chemotherapy arm received subsequent immunotherapy. One-third of patients had PD-L1 expression $<1 \%$, squamous histology subtype, and were females. Nivolumab plus ipilimumab in high TMB was associated with longer PFS (7.2 vs 5.5 months, HR $0.58 ; 97.5 \%$ CI, 0.41 to $0.81 ; p<0.001)$ and increased RR (45\% vs $27 \%$ ) compared with chemotherapy. The PFS benefit was observed for all TMB-high subgroups regardless of PD-L1 expression ( $\geq 1 \%$, HR: $0.62(95 \% \mathrm{CI}$, $0.44-0.88$ ) or $<1 \%$, HR 0.48 (95\% CI, 0.27-0.85)); however, the percentage of patients with tumors with PD$\mathrm{L} 1 \geq 50 \%$ is not reported; histologic subtype (squamous, HR 0.63 95\%CI, [0.39-1.04]; nonsquamous, HR 0.55; 95\%CI [0.38-0.80]), sex (male, HR 0.52; 95\%CI [0.36-0.74]; female HR 0.70; 95\%CI [0.41-1.20]), and performance status (PS 0, HR 0.62; 95\%CI [0.38-1.02]; PS 1 , HR 0.55 ; 95\%CI [0.38-0.80]). It is noteworthy that PFS curves cross during the first four months of treatment, suggesting that selected patients according to high TMB criteria may not overcome the risk of rapid progression or lack of response with immunotherapy combination. The rate of grade $\geq 3$ treatment-related AEs was $31.2 \%$ with immune combination vs $36.1 \%$ with chemotherapy, mainly hepatic events. However, a higher proportion of patients discontinued treatment as a consequence of AEs in nivolumab and ipilimumab arm than in the chemotherapy arm $(17.4 \% \text { vs } 8.9 \%)^{9}$

A recent press release about a CheckMate 227 trial on 19 October $2018^{52}$ reported an updated descriptive analysis showing that the HR for OS with nivolumab and ipilimumab versus chemotherapy in patients with $\mathrm{TMB} \geq 10 \mathrm{Mut} /$ $\mathrm{Mb}$ was 0.77 (95\% CI: 0.56-1.06), with a median OS of 23.3 months and 16.7 months, respectively. Of note, among patients with $\mathrm{TMB}<10 \mathrm{Mut} / \mathrm{Mb}$ the HR for OS (HR 0.78, 95\% CI 0.61-1.00) was comparable to that observed in patients with high $\mathrm{TMB},{ }^{52}$ suggesting a potential prognostic value rather predictive value for high TMB. Based on this lack of statistically significant benefit in OS, the company believes further evidence on the relationship between TMB 
and PD-L1 is required to fully evaluate the impact of nivoluamb plus ipilimumab on OS in first-line NSCLC patients with high TMB, and the sponsor has withdrawn regulatory applications for lung cancer drug combination with nivolumab and ipilimumab in this population.

Approximately one-third of NSCLC tumors do not express PD-L1. In the first-line setting, in PD-L1 negative tumors, the combination of pembrolizumab and chemotherapy significantly improves the outcome compared with chemotherapy regardless of the histological subtype., ${ }^{2,4}$ One secondary endpoint in the CheckMate 227 trial was to assess in first-line setting the efficacy of nivolumab in combination either with ipilimumab $(\mathrm{N}=187)$ or with chemotherapy $(\mathrm{N}=177)$ and compared with chemotherapy alone $(\mathrm{N}=186)$ in PD-L1 negative NSCLC patients. In patients with $\mathrm{TMB} \geq 10 \mathrm{Mut} / \mathrm{Mb}$ and $\mathrm{PD}-\mathrm{L} 1$ expression $<1 \%$, the PFS was longer with nivolumab in combination with either chemotherapy (6.2 months vs 5.3 months, HR $0.56,95 \%$ CI $0.35-0.91)$ or ipilimumab (7.7 months vs 5.3 months, HR $0.48,95 \%$ CI $0.27-0.85)$ compared with chemotherapy alone; the RR was also higher ( $61 \%$ vs $37 \%$ and $21 \%$, respectively). Conversely, in the subset of patients with $<10 \mathrm{Mut} / \mathrm{Mb}$ and $<1 \%$ PD-L1 expression, the addition of nivolumab to either chemotherapy or ipilimumab did not appear to have any PFS benefit in comparison with chemotherapy alone (HR $0.87,95 \%$ CI $0.57-1.33$ for nivolumab plus chemotherapy and $\mathrm{HR} 1.17,95 \% \mathrm{CI} 0.76-1.81$ for nivolumab-ipilimumab combination). ${ }^{53}$ These results could suggest that TMB may play a role in selecting negative PDL1 NSCLC patients suitable for receiving immune checkpoint inhibitors. However, contrary to other trials, ${ }^{2,4}$ no survival data have been reported; therefore, it remains unknown whether or not TMB should be used in PD-L1 negative patients as a predictive marker. The outcome, the treatment cost, and the safety may help to elucidate the best upfront strategy in this population.

Other ongoing first-line clinical trials are assessing the role of nivolumab and ipilimumab with or without chemotherapy (Table 3), such as the multi-cohort phase III/IV CheckMate 877 trial (NCT02869789), ${ }^{54}$ and the CheckMate 9LA trial (NCT03215706), as well as the MYSTIC trial assessing the role of durvalumab and tremelimumab in this scenario. Recent data have reported comparable exposure, safety and efficacy between flat dose nivolumab compared to the weight-based regimen. ${ }^{55,56}$ This schedule is being prospectively evaluated in the multi-cohort CheckMate 817 phase IIIB/IV trial, which assesses the efficacy of flat dose nivolumab
(240 mg Q2W) plus ipilimumab (1 mg/kg Q6W) until progression in PD-L1 positive or negative, chemotherapy naive NSCLC patients (arm A). Other cohorts included in the CheckMate 817 trial were: cohort A1 testing the combination in special populations (HIV patients, patients with BM, and patients with renal or hepatic failure), cohort B testing the combination in previously treated patients with at least one platinum-based chemotherapy, and cohort $\mathrm{C}$ enrolling patients whose tumors have high TMB ( $\geq 10 \mathrm{Mut} / \mathrm{Mb}$ ). The primary endpoint of the trial is the proportion grade $\geq 3$ of treatmentrelated AEs (TR-AEs) and ir-AEs. Secondary endpoints include RR, duration of response (DoR), PFS, and OS. For instance, only results from cohort A, enrolling 391 treatment naive patients, have been reported. The majority of patients in this cohort were current or former smokers (91\%) and had an adenocarcinoma lung cancer $(72 \%)$. PD-L $1 \geq 1 \%$ expression (tested by $28-8$ IHC) was reported in $49 \%$ of patients, including $18 \%$ of patients with PD-L1 expression $\geq 50 \%$. The TMB (by FoundationOne CDx assay) was evaluable in $39 \%$ of patients $(\mathrm{N}=151)$ and among these patients, $48 \%$ had high TMB. All grades of TR-AEs occurred in $75 \%$ of patients, with $32 \%$ of grade $\geq 3$ TR-AEs leading to treatment discontinuation in $18 \%$ of cases. The median time to onset TR-selected AEs ranged from 2.1 to 19.2 weeks. The median PFS was 6 months, and median RR was 35\%, the without differences according to histologic subtype. For efficacy in biomarker-selected patients, better RR and PFS were reported in PD-L $1 \geq 1 \%$ verses $\mathrm{PD}-\mathrm{L} 1<1 \%$ tumors ( $41 \%$ vs $28 \%$ and 8.1 vs 5.3 months), as well as in TMB high versus low tumors (54\% vs $29 \%$ and 10.9 vs 4.2 months). ${ }^{54}$ These results report comparable clinical activity (PFS and RR) in patients with high TMB across CheckMate 227, 568 and 817 (Table 2).

The randomized phase III MYSTIC trial assessed the efficacy of durvalumab (20 mg/kg Q4W) monotherapy or durvalumab and tremelimumab (D: $20 \mathrm{mg} / \mathrm{kg}$ Q4W, T: $1 \mathrm{mg} / \mathrm{kg}$ Q4W up to four doses) compared with standard of care platinum-based chemotherapy in 1118 immunotherapy- and chemotherapy-naive stage IV NSCLC patients. Primary endpoints were assessed in patients with PD-L1 expression $\geq 25 \%$ (by SP263 IHC, N=488) and were OS for durvalumab versus chemotherapy, and OS and PFS for durvalumab and tremelimumab arm versus chemotherapy. Both co-primary PFS and OS endpoints were not met for either durvalumab monotherapy (PFS: 4.7 vs 5.4 months, HR 0.87; 99.5\% CI, 0.593-1.285; 
Table 2 Efficacy of nivolumab and ipilimumab as first-line treatment in advanced NSCLC patients with high TMB

\begin{tabular}{|c|c|c|c|c|c|}
\hline Study & $\mathbf{N}$ & $\begin{array}{l}\text { RR } \\
\text { (\%) }\end{array}$ & DoR (mo.) & $\begin{array}{l}\text { PFS } \\
\text { (mo) }\end{array}$ & $\begin{array}{l}\text { OS } \\
\text { (mo.) }\end{array}$ \\
\hline $\begin{array}{l}\text { CheckMate } \\
012^{51}\end{array}$ & 24 & 51 & Not reported & 17.1 & $\begin{array}{l}\text { Not } \\
\text { reported }\end{array}$ \\
\hline $\begin{array}{l}\text { CheckMate } \\
568^{14}\end{array}$ & 49 & 44 & Not reported & 7.2 & $\begin{array}{l}\text { Not } \\
\text { reported }\end{array}$ \\
\hline $\begin{array}{l}\text { CheckMate } \\
227^{9}\end{array}$ & 139 & 45.3 & $\begin{array}{l}\text { Not reached. } \\
\text { I-year DoR: } 68 \%\end{array}$ & 7.2 & 23.3 \\
\hline $\begin{array}{l}\text { CheckMate } \\
817^{* 54}\end{array}$ & 73 & 54 & Not reported & 10.9 & $\begin{array}{l}\text { Not } \\
\text { reported }\end{array}$ \\
\hline
\end{tabular}

Abbreviations: DoR, duration of response. PFS, progression-free survival. OS, overall survival; mo., months; TMB, tumor mutation burden.*Cohort A.

Table 3 Ongoing clinical trials in the first-line setting with nivolumab and ipilimumab

\begin{tabular}{|c|c|c|}
\hline NCT & Phase & Treatment \\
\hline NCT03377023 & 1 & $\begin{array}{l}\text { Nivolumab and ipilimumab and } \\
\text { nintedanib }\end{array}$ \\
\hline NCT02696993 & $\mathrm{I} / \mathrm{II}$ & $\begin{array}{l}\text { Nivolumab } \pm \text { Ipilimumab and RT in brain } \\
\text { MI }\end{array}$ \\
\hline NCT02983045 & $\mathrm{I} / \mathrm{II}$ & Nivolumab + NKTR-2I4士 ipilimumab \\
\hline NCT03573947 & II & Nivolumab and ipilimumab and paclitaxel \\
\hline NCT0342533I & II & Nivolumab and ipilimumab \\
\hline NCT0300I882 & II & $\begin{array}{l}\text { Nivolumab and ipilimumab (CheckMate } \\
592 \text { ) }\end{array}$ \\
\hline NCT032I5706 & III & $\begin{array}{l}\text { Nivolumab and ipilimumab and che- } \\
\text { motherapy (CheckMate 9LA) }\end{array}$ \\
\hline NCT03469960 & III & Nivolumab and ipilimumab (DICIPLE) \\
\hline
\end{tabular}

$p=0.324$; OS: 16.3 vs 12.9 months, HR $0.76,97.54 \%$ CI $0.564-1.019$; nominal $p=0.036$ ) or durvalumab and tremelimumab (PFS: 3.9 vs 5.4 months, HR 1.05; 99.5\% CI, $0.722,1.534 ; p=0.705$ and OS: 11.9 vs 12.9 months HR $0.85,98.77 \%$ CI $0.611-1.173$; nominal $p=0.202$ ) compared with chemotherapy. These results do not currently support the use of durvalumab as a single agent or in combination with tremelimumab in this patient population. The ongoing randomized phase III trial NEPTUNE (NCT02542293) is assessing durvalumab and tremelimumab vs chemotherapy.

An exploratory analysis of the MYSTIC trial ${ }^{15}$ examined the OS according to high blood (b)TMB ( $\geq 16 \mathrm{Mut} / \mathrm{Mb})$. More than $70 \%$ of patients had bTMB evaluation, $40 \%$ of whom had high $\mathrm{bTMB}$; for these patients OS was 16.5 months with the combination of durvalumab and tremelimumab versus 10.5 months with chemotherapy (HR 0.62; 95\%CI: 0.45-0.86), but durvalumab monotherapy did not improve the OS compared with chemotherapy (11 vs 10.5 months, HR $0.80 ; 95 \%$ CI: $0.59-1.07)$. The 2-year OS in high bTMB was $39 \%$ with the combination, $30 \%$ with durvalumab and $18 \%$ with chemotherapy. For patients with low bTMB, no survival differences were reported between arms, and median OS was 8.5 months with durvalumab and tremelimumab, 12.2 months with durvalumab alone and 11.6 months with chemotherapy. ${ }^{15}$ Similarly, in a recent exploratorty analysis with $\mathrm{bTMB} \geq 20 \mathrm{mut} / \mathrm{Mb}$ survival benefit has been reported with durvalumab and tremelimumab compared with chemotherapy. ${ }^{15}$ Contrary to CheckMate 227, results from the MYSTIC trial show a potential role for TMB as a predictive biomarker for survival benefit from a combination of immunotherapies; however, it was an exploratory analysis and these results would require prospective validation.

At least four randomized phase III clinical trials have reported that the combination of anti-PD(L)1 and chemotherapy with ${ }^{57}$ or without bevacizumab ${ }^{2,4,58}$ improved the OS compared with standard of care regardless of histology and PD-L1 expression. The purpose of the ongoing phase III CheckMate 9LA trial (NCT03215706) is to determine whether nivolumab plus Ipilimumab plus chemotherapy improves the OS compared with chemotherapy alone in the first-line setting. Similarly, the randomized phase III POSEIDON trial (NCT03164616) evaluates durvalumab plus chemotherapy with or without tremelimumab or chemotherapy alone in the same population. Patients are not selected according to PD-L1 expression or high TMB in any of both trials. For instance, different first-line strategies are available; therefore, the magnitude of benefit according to validated and accepted scales $^{59}$ and toxicity profile may help to elucidate the best treatment approach.

\section{Other clinical questions}

\section{Patients with brain metastases}

Up to $30 \%$ of advanced NSCLC patients have BM at baseline, with a 3 -year OS $<10 \%,{ }^{60}$ suggesting that new systemic treatment options are eagerly awaited. In a series of matched BM and primary NSCLC samples, PD-L1 positive samples ( $\geq 5 \%$ with E1L3N antibody) was lower for the BM compared to the paired primary ( 33 vs $44 \%$ ). ${ }^{61}$ Conversely, TMB was significantly higher in the BM specimens. ${ }^{62}$ This observation may suggest a potentially differing RR with ICI in BM compared with the primary 
tumor. The majority of clinical trials exclude patients with $\mathrm{BM}$; however, retrospective data, ${ }^{63}$ expanded access programs cohorts, ${ }^{64,65}$ and data from randomized phase III clinical trials in pre-treated advanced NSCLC patients ${ }^{66}$ suggest that central nervous system (CNS) involvement does not negatively impact the outcome on ICI, although CNS failure is more common in patients with BM at baseline compared to those without $\mathrm{BM}$ at baseline. ${ }^{63}$ For monotherapy with ICI there has been activity reported in a phase II trial enrolling melanoma and lung cancer patients with BM. For pembrolizumab an intracranial RR of $33 \%$ was reported in 18 PD-L1-positive NSCLC patients with untreated $(\mathrm{N}=8)$ or progressive asymptomatic $\mathrm{BM}$ between 5 and $20 \mathrm{~mm}$ in diameter, with a duration of response of more than 6 months. Neurological AEs were uncommon and manageable without grade 3 AEs. ${ }^{67}$ In the randomized phase III trials in first-line (KEYNOTE 189 $(\mathrm{N}=108),{ }^{2}$ KEYNOTE $\left.024(\mathrm{~N}=28)\right)^{1}$ or second-line treatment (OAK trial $(\mathrm{N}=203)),{ }^{8}$ patients with $\mathrm{BM}$ were enrolled, and BM did not negatively impact in the outcome with ICI. The combination of nivolumab and ipilimumab has shown the most impressive CNS response rates to immunotherapy in two phase II trials. ${ }^{68,69}$ In the phase II CheckMate 204 study, 94 metastatic melanoma patients given nivolumab plus ipilimumab for up to four doses, followed by nivolumab until progression, reported an intracranial RR of $55 \%$, with a treatment-related $\geq$ grade 3 AEs in $55 \%$ of patients. Five patients (5\%) discontinued therapy due to immune-related neurologic adverse events. ${ }^{68}$ These results were confirmed in the second phase II trial ${ }^{69}$ in 36 patients with metastatic melanoma. Both trials suggest the activity and safety of nivolumab and ipilimumab in patients with untreated and asymptomatic BM. However, combination ICI treatment in NSCLC patients with BM is scarce. In the CheckMate 227 trial patients with $\mathrm{BM}$ were eligible if they were adequately treated and did not report neurological symptoms 2 weeks before randomization. However, efficacy in this population has not been reported.

For instance, it remains unknown to what extent the therapeutic effect of ICIs in BM relies on the local activity of ICI in the primary tumor or whether the use of previous radiotherapy may help to enhance the central activity of ICIs, ${ }^{70}$ as well as whether ICI combination strategies might also increase brain activity. The ongoing NCT02696993 trial and the CheckMate 817 trial (NCT 02869789, arm A1) are addressing these questions. Another challenge is to establish the place of RT with ICI. There is preclinical ${ }^{71}$ and clinical ${ }^{72,73}$ evidence suggesting that the concurrent strategy may have synergistic activity and favorable survival outcome compared with the sequential strategy; however, weighed activity and toxicity, mainly radionecrosis, ${ }^{74,75}$ should be carefully evaluated. Different clinical trials are ongoing about the efficacy of radiotherapy and ICI, and results of some of these trials have been summarized in a recent review. ${ }^{76}$

\section{Elderly population}

The elderly population, patients older than 70 years, are underrepresented in clinical trials, and represent a low proportion of all study populations in some studies. The median age of patients enrolled in clinical trials assessing nivolumab and ipilimumab was 68 years in CheckMate $012,{ }^{46} 66$ years in CheckMate $058,{ }^{77} 65$ years in CheckMate 817 with $53 \%$ of patients older than $65,{ }^{54}$ and 64 years in the phase III CheckMate 227 trial, which includes $38 \%$ of patients between 65 and 75 years and $9.4 \%$ of patients older than 75 years. ${ }^{9}$ It has been suggested that elderly patients may derive less benefit from $\mathrm{ICI}^{78}$ or may have increased risk of hyper-progressive disease on treatment, ${ }^{79}$ but this association between age and risk of hyper-progressive disease has not been observed in a cohort of NSCLC patients. ${ }^{80}$ It has been suggested that the benefit of ICI is more related to the "age" of the immune system (immunosenescence) rather than patients' age. The immunosenescence phenotype occurs in one-third of NSCLC patients, and it is independent of age and correlates with lower disease control rate in NSCLC patients treated with anti-PD(L)1 treatment. ${ }^{81}$ The HR for PFS according to age in the CheckMate 227 trial was $0.51(0.34-0.77), 0.62$ $(0.4-0.97)$ and $0.42(0.14-1.30)$, for patients of $\leq 65$ years, from 65 to 75 years and $\geq 75$ years, respectively. However, the limited number of patients older than 75 years $(\mathrm{N}=27)$ does not lead to any firm conclusions. As the elderly population may have more comorbidities, toxicity and efficacy ratio must be weighted as well as quality of life before the broad acceptance of nivolumab plus ipilimumab combination regardless of age. Specific clinical trials in elderly populations are awaited.

\section{PD-LI expression $\geq 50 \%$}

One clinical question is what is the best treatment strategy in NSCLC patients with PD-L1 expression $\geq 50 \%$, monotherapy with pembrolizumab, ${ }^{1,82}$ combination strategy of ICI with chemotherapy with or without bevacizumab, ${ }^{2-4}$ or nivolumab and ipilimumab combination. ${ }^{9}$ Although TMB 
and PD-L1 are independent biomarkers, in the CheckMate 026 trial approximately $10 \%$ of patients shared both biomarkers (high TMB and PD-L1 $\geq 50 \%$ ) and those patients derived the maximum benefit of nivolumab. ${ }^{13}$ In the KEYNOTE 024 trial, pembrolizumab in PD-L1 $\geq 50 \%$ reported a RR of $45 \%$, median PFS and OS of 10 months and 30 months, respectively, with 1 -year OS of $70 \%$ despite $54 \%$ of crossover to ICI in the control arm. ${ }^{1,82}$ In the CheckMate 227 trial $^{9}$ nivolumab and ipilimumab in high TMB reported similar outcomes (RR of $45 \%$, PFS of 7.1 months, and 1-year of OS 67\%). However, the efficacy has not been reported according to PD-L1 expression strata, crossover was not allowed, and contrary to the KEYNOTE 024 trial, there is no survival benefit with a combination of nivolumab and ipilimumab and PFS curves in the CheckMate 227 trial crosses, not reinforcing that double immunotherapy strategy is better than monotherapy in this subgroup of NSCLC patients. The ongoing phase III KEYNOTE 589 trial (NCT03302234) may in part answer this question. The trial randomizes patients with PD-L1 $\geq 50 \%$ to pembrolizumab and ipilimumab versus pembrolizumab and placebo. However, TMB is not a stratification criterion.

\section{Treatment duration}

Treatment duration is a challenge in ICI strategy. Indeed, NSCLC patients treated with atezolizumab ${ }^{83}$ or nivolumab $^{84}$ achieve long-term survival despite discontinuing treatment for ir-AEs and receiving corticosteroids, but it remains unknown whether this survival benefit is linked to the time of treatment exposure or to the onset of ir-AEs, which correlates with a better outcome in NSCLC patients. ${ }^{85-94}$ In previously treated NSCLC patients the CheckMate 153 trial $^{95}$ suggests that stopping nivolumab treatment after one year in the case of clinical and radiological benefit could be harmful, with shorter PFS compared with prolonged treatment beyond one year. In the melanoma, a treatment of 6 months of ipilimumab demonstrated its efficacy. The objective of the randomized phase III DICIPLE trial (NCT03469960) is to demonstrate that a treatment of 6 months of nivolumab plus ipilimumab followed by an observation (stop and go) is not less effective than nivolumab plus ipilimumab treatment given until progression or toxicity. This strategy would allow the accumulated toxicities to decrease, improve the quality of life and decrease the costs. The primary endpoint is PFS and 1,360 advanced treatment-naive PD-L1 $\geq 1 \%$ but $<50 \%$ NSCLC patients will be enrolled.

\section{Conclusion}

Next generation sequencing is increasing in daily clinical practice and in consequence so is the determination of TMB. However, TMB can only be assessed in half of NSCLC patients and just one-third has high TMB. Assays and definitions for high TMB should be standardized in both tissue and blood. Indeed, the real predictive value of high TMB over its prognostic value merits further evaluation. Despite the efficacy of nivolumab plus ipilimumab in selected patients according to the highTMB, compared with other strategies in the first-line setting, no survival benefit has been reported with the combination of nivolumab and ipilimumab; some patients may present hyper-progressive disease on treatment despite being selected for a predictive biomarker, and the cost of the potential biomarker for this combination is higher than PD-L1 immunohistochemistry expression as well as the toxicity profile. These limitations may limit broad acceptance of this combination in the current strategy of advanced NSCLC patients.

\section{Disclosure}

The authors report no conflicts of interest in this work.

\section{References}

1. Reck M, Rodríguez-Abreu D, Robinson AG, et al. Pembrolizumab versus chemotherapy for PD-L1-positive non-small-cell lung cancer. $N$ Engl $J$ Med. 2016;375(19):1823-1833. doi:10.1056/NEJMoa 1606774

2. Gandhi L, Rodríguez-Abreu D, Gadgeel S, et al. Pembrolizumab plus chemotherapy in metastatic non-small-cell lung cancer. $N$ Engl J Med. 2018;378(22):2078-2092. doi:10.1056/NEJMoa1801005

3. Socinski MA, Jotte RM, Cappuzzo F, et al. Overall survival (OS) analysis of IMpower150, a randomized $\mathrm{Ph} 3$ study of atezolizumab (atezo) + chemotherapy (chemo) \pm bevacizumab (bev) vs chemo + bev in 1L nonsquamous (NSQ) NSCLC. JCO. 2018;36(15_suppl):9002. doi:10.1200/JCO.2018.36.15_suppl.9002

4. Paz-Ares L, Luft A, Vicente D, et al. Pembrolizumab plus chemotherapy for squamous non-small-cell lung cancer. $N$ Engl J Med. 2018;379 (21):2040-2051. doi:10.1056/NEJMoa1810865

5. Herbst RS, Baas P, Kim D-W, et al. Pembrolizumab versus docetaxel for previously treated, PD-L1-positive, advanced non-small-cell lung cancer (KEYNOTE-010): a randomised controlled trial. Lancet. 2016;387(10027):1540-1550. doi:10.1016/S0140-6736(15)01281-7

6. Borghaei H, Paz-Ares L, Horn L, et al. Nivolumab versus docetaxel in advanced nonsquamous non-small-cell lung cancer. $N$ Engl $J$ Med. 2015;373(17):1627-1639. doi:10.1056/NEJMoa1507643

7. Brahmer J, Reckamp KL, Baas P, et al. Nivolumab versus docetaxel in advanced squamous-cell non-small-cell lung cancer. $N$ Engl $J$ Med. 2015;373(2):123-135. doi:10.1056/NEJMoa1504627

8. Fehrenbacher L, von Pawel J, Park K, et al. Updated efficacy analysis including secondary population results for OAK: a randomized phase III study of atezolizumab versus docetaxel in patients with previously treated advanced non-small cell lung cancer. J Thorac Oncol. 2018;13 (8):1156-1170. doi:10.1016/j.jtho.2018.04.039 
9. Hellmann MD, Ciuleanu T-E, Pluzanski A, et al. Nivolumab plus ipilimumab in lung cancer with a high tumor mutational burden. $N$ Engl J Med. 2018;378(22):2093-2104. doi:10.1056/NEJMoa180 1946

10. Shien K, Papadimitrakopoulou VA, Wistuba II. Predictive biomarkers of response to PD-1/PD-L1 immune checkpoint inhibitors in non-small cell lung cancer. Lung Cancer. 2016;99:79-87. doi:10.1016/j.lungcan.2016.06.016

11. Herzberg B, Campo MJ, Gainor JF. Immune checkpoint inhibitors in non-small cell lung cancer. Oncologist. 2017;22(1):81-88. doi:10.1634/theoncologist.2016-0189

12. Khunger M, Hernandez AV, Pasupuleti V, et al. Programmed cell death 1 (PD-1) ligand (PD-L1) expression in solid tumors as a predictive biomarker of benefit from PD-1/PD-L1 axis inhibitors: a systematic review and meta-analysis. JCO Precis Oncol. 2017; (1):1-15. doi:10.1200/PO.16.00030

13. Carbone DP, Reck M, Paz-Ares L, et al. First-line nivolumab in stage IV or recurrent non-small-cell lung cancer. $N$ Engl J Med. 2017;376 (25):2415-2426. doi:10.1056/NEJMoa1613493

14. Ready N, Hellmann MD, Awad MM, et al. First-line nivolumab plus Ipilimumab in advanced non-small-cell lung cancer (CheckMate 568): outcomes by programmed death ligand 1 and tumor mutational burden as biomarkers. J Clin Oncol. 2019:JCO1801042. 10.1200/ JCO.18.01042.

15. Rizvi NA, Chul Cho B, Reinmuth N, et al. Durvalumab with or without tremelimumab vs platinum-based chemotherapy as first-line treatment for metastatic non-small cell lung cancer: MYSTIC. Ann Oncol. 2018;29(suppl_10):mdy511.005-mdy511.005. doi:10.1093/ annonc/mdy511.005

16. Hendriks LE, Rouleau E, Besse B. Clinical utility of tumor mutational burden in patients with non-small cell lung cancer treated with immunotherapy. Transl Lung Cancer Res. 2018;7(6):647-660. doi:10.21037/tlcr.2018.09.22

17. Chan TA, Yarchoan M, Jaffee E, et al. Development of tumor mutation burden as an immunotherapy biomarker: utility for the oncology clinic. Ann Oncol. 2019;30(1):44-56. doi:10.1093/annonc/mdy495

18. Coulie PG, Van Den Eynde BJ, van der Bruggen P, Boon T. Tumour antigens recognized by $\mathrm{T}$ lymphocytes: at the core of cancer immunotherapy. Nat Rev Cancer. 2014;14(2):135-146. doi:10.1038/ $\operatorname{nrc} 3670$

19. Rizvi H, Sanchez-Vega F, La K, et al. Molecular determinants of response to anti-programmed cell death (PD)-1 and anti-programmed death-ligand 1 (PD-L1) blockade in patients with non-small-cell lung cancer profiled with targeted next-generation sequencing. $J$ Clin Oncol. 2018;36(7):633-641. doi:10.1200/JCO.2017.75.3384

20. Chaudhary R, Quagliata L, Martin JP, et al. A scalable solution for tumor mutational burden from formalin-fixed, paraffin-embedded samples using the oncomine tumor mutation load assay. Transl Lung Cancer Res. 2018;7(6):616-630. doi:10.21037/tlcr.2018. 08.01

21. Camidge DR, Doebele RC, Kerr KM. Comparing and contrasting predictive biomarkers for immunotherapy and targeted therapy of NSCLC. Nat Rev Clin Oncol. 2019. doi:10.1038/s41571-019-0173-9

22. McGranahan N, Furness AJS, Rosenthal R, et al. Clonal neoantigens elicit $\mathrm{T}$ cell immunoreactivity and sensitivity to immune checkpoint blockade. Science. 2016;351(6280):1463-1469. doi:10.1126/science. aaf1490

23. Samstein RM, Lee C-H, Shoushtari AN, et al. Tumor mutational load predicts survival after immunotherapy across multiple cancer types. Nat Genet. 2019;51(2):202-206. doi:10.1038/s41588-018-0312-8

24. Lawrence MS, Stojanov P, Polak P, et al. Mutational heterogeneity in cancer and the search for new cancer-associated genes. Nature. 2013;499(7457):214-218. doi:10.1038/nature12213

25. Zehir A, Benayed R, Shah RH, et al. Mutational landscape of metastatic cancer revealed from prospective clinical sequencing of 10,000 patients. Nat Med. 2017;23(6):703-713. doi:10.1038/nm.4333
26. Rizvi NA, Hellmann MD, Snyder A, et al. Cancer immunology. Mutational landscape determines sensitivity to PD-1 blockade in non-small cell lung cancer. Science. 2015;348(6230):124-128. doi: $10.1126 /$ science.aaa 1348

27. Robinson DR, Wu Y-M, Lonigro RJ, et al. Integrative clinical genomics of metastatic cancer. Nature. 2017;548(7667):297-303. doi:10.1038/nature23306

28. Fabrizio D, Lieber D, Malboeuf C, et al. A blood-based next-generation sequencing assay to determine tumor mutational burden (bTMB) is associated with benefit to an anti-PD-L1 inhibitor, atezolizumab. Cancer Res. 2018;78(13Supplement):5706. doi:10.1158/1538-7445.AM2018-5706

29. Gandara DR, Paul SM, Kowanetz M, et al. Blood-based tumor mutational burden as a predictor of clinical benefit in non-small-cell lung cancer patients treated with atezolizumab. Nat Med. 2018;24 (9):1441-1448. doi:10.1038/s41591-018-0134-3

30. Kim ES, Velcheti V, Mekhail T, et al. Primary efficacy results from B-F1RST, a prospective phase II trial evaluating blood-based tumour mutational burden (bTMB) as a predictive biomarker for atezolizumab (atezo) in 1L non-small cell lung cancer (NSCLC). Ann Oncol. 2018;29(suppl_8):mdy424.067-mdy424.067. doi:10.1093/annonc/ mdy424.067

31. Das R, Verma R, Sznol M, et al. Combination therapy with anti-CTLA-4 and anti-PD-1 leads to distinct immunologic changes in vivo. J Immunol. 2015;194(3):950-959. doi:10.4049/jimmunol.1401686

32. Wei SC, Levine JH, Cogdill AP, et al. Distinct cellular mechanisms underlie anti-CTLA-4 and anti-PD-1 checkpoint blockade. Cell. 2017;170(6):1120-1133.e17. doi:10.1016/j.cell.2017.07.024

33. Le DT, Durham JN, Smith KN, et al. Mismatch repair deficiency predicts response of solid tumors to PD-1 blockade. Science. 2017;357(6349):409-413. doi:10.1126/science.aan6733

34. Bonneville R, Krook MA, Kautto EA, et al. The landscape of microsatellite instability across 39 cancer types. JCO Precis Oncol. 2017;1:1-15. doi:10.1200/PO.17.00073

35. Zaretsky JM, Garcia-Diaz A, Shin DS, et al. Mutations associated with acquired resistance to PD-1 blockade in melanoma. $N$ Engl J Med. 2016;375(9):819-829. doi:10.1056/NEJMoa1604958

36. Riaz N, Havel JJ, Kendall SM, et al. Recurrent SERPINB3 and SERPINB4 mutations in patients who respond to anti-CTLA4 immunotherapy. Nat Genet. 2016;48(11):1327-1329. doi:10.1038/ ng.3677

37. Banerjee T, Duhadaway JB, Gaspari P, et al. A key in vivo antitumor mechanism of action of natural product-based brassinins is inhibition of indoleamine 2,3-dioxygenase. Oncogene. 2008;27(20):2851-2857. doi:10.1038/sj.onc.1210939

38. Mariathasan S, Turley SJ, Nickles D, et al. TGF $\beta$ attenuates tumour response to PD-L1 blockade by contributing to exclusion of T cells. Nature. 2018;554(7693):544-548. doi:10.1038/nature25501

39. Cristescu R, Mogg R, Ayers M, et al. Pan-tumor genomic biomarkers for PD-1 checkpoint blockade-based immunotherapy. Science. 2018;362(6411):eaar3593. doi:10.1126/science.aar3593

40. Auslander N, Zhang G, Lee JS, et al. Robust prediction of response to immune checkpoint blockade therapy in metastatic melanoma. Nat Med. 2018;24(10):1545-1549. doi:10.1038/s41591-018-0157-9

41. Jiang $\mathrm{P}, \mathrm{Gu} \mathrm{S}, \mathrm{Pan} \mathrm{D}$, et al. Signatures of $\mathrm{T}$ cell dysfunction and exclusion predict cancer immunotherapy response. Nat Med. 2018;24 (10):1550-1558. doi:10.1038/s41591-018-0136-1

42. Roy S, Trinchieri G. Microbiota: a key orchestrator of cancer therapy. Nat Rev Cancer. 2017;17(5):271-285. doi:10.1038/nrc.2017.13

43. Routy B, Le Chatelier E, Derosa L, et al. Gut microbiome influences efficacy of PD-1-based immunotherapy against epithelial tumors. Science (New York, NY). 2018;359(6371):91-97. doi:10.1126/science.aan3706

44. Gopalakrishnan V, Spencer CN, Nezi L, et al. Gut microbiome modulates response to anti-PD-1 immunotherapy in melanoma patients. Science (New York, NY). 2018;359(6371):97-103. doi: $10.1126 /$ science.aan 4236 
45. Gettinger S, Beck T, Yang X, et al. CheckMate 592: a phase II exploratory study of biomarkers associated with the efficacy of first-line nivolumab (nivo) plus ipilimumab (ipi) in patients (pts) with stage IV or recurrent NSCLC. Ann Oncol. 2018;29(suppl_8): mdy292.124-mdy292.124. doi:10.1093/annonc/mdy292.124

46. Hellmann MD, Rizvi NA, Goldman JW, et al. Nivolumab plus ipilimumab as first-line treatment for advanced non-small-cell lung cancer (CheckMate 012): results of an open-label, phase 1, multicohort study. Lancet Oncol. 2017;18(1):31-41. doi:10.1016/S1470-2045(16)30624-6

47. Rizvi NA, Hellmann MD, Brahmer JR, et al. Nivolumab in combination with platinum-based doublet chemotherapy for first-line treatment of advanced non-small-cell lung cancer. J Clin Oncol. 2016;34 (25):2969-2979. doi:10.1200/JCO.2016.66.9861

48. Juergens R, Hellmann M, Brahmer J, et al. First-line nivolumab plus platinum-based doublet chemotherapy for advanced NSCLC: CheckMate 012 3-year update. J Thorac Oncol. 2017;12(11): S1792-S1793. doi:10.1016/j.jtho.2017.09.429

49. Gettinger S, Rizvi NA, Chow LQ, et al. Nivolumab monotherapy for first-line treatment of advanced non-small-cell lung cancer. J Clin Oncol. 2016;34(25):2980-2987. doi:10.1200/JCO.2016.66.9929

50. Goldman JW, Antonia SJ, Gettinger SN, et al. Nivolumab (N) plus ipilimumab (I) as first-line (1L) treatment for advanced (adv) NSCLC: 2-yr OS and long-term outcomes from CheckMate 012. JCO. 2017;35 (15_suppl):9093. doi:10.1200/JCO.2017.35.15_suppl.9093

51. Hellmann MD, Nathanson T, Rizvi H, et al. Genomic features of response to combination immunotherapy in patients with advanced non-small-cell lung cancer. Cancer Cell. 2018;33(5):843-852.e4. doi:10.1016/j.ccell.2018.03.018

52. Bristol-Myers Squibb. (2018). Bristol-Myers Squibb provides update on the ongoing regulatory review of opdivo plus low-dose yervoy in first-line lung cancer patients with tumor mutational burden $\geq 10$ mut/ Mb. [online] Available from: https://news.bms.com/press-release/corporatefinancial-news/bristol-myers-squibb-provides-update-ongoingregulatory-review. Accessed December 20, 2018.

53. Borghaei H, Hellmann MD, Paz-Ares LG, et al. Nivolumab (Nivo) + platinum-doublet chemotherapy (Chemo) vs chemo as first-line (1L) treatment (Tx) for advanced non-small cell lung cancer (NSCLC) with $<1 \%$ tumor PD-L1 expression: results from CheckMate 227. JCO. 2018;36(15_suppl):9001. doi:10.1200/JCO.2018.36.15_suppl.9001

54. Paz-Ares L, Urban L, Audigier-Valette C, et al. CheckMate 817: safety of flat-dose nivolumab plus weight-based ipilimumab for the first-line (1L) treatment of advanced NSCLC. J Thorac Oncol. 2018;13(10):S493. doi:10.1016/j.jtho.2018.08.635

55. Zhao X, Suryawanshi S, Hruska M, et al. Assessment of nivolumab benefit-risk profile of a $240-\mathrm{mg}$ flat dose relative to a $3-\mathrm{mg} / \mathrm{kg}$ dosing regimen in patients with advanced tumors. Ann Oncol. 2017;28 (8):2002-2008. doi:10.1093/annonc/mdx235

56. Long GV, Tykodi SS, Schneider JG, et al. Assessment of nivolumab exposure and clinical safety of $480 \mathrm{mg}$ every 4 weeks flat-dosing schedule in patients with cancer. Ann Oncol. 2018;29(11):2208-2213. doi:10.1093/annonc/mdy408

57. Socinski MA, Jotte RM, Cappuzzo F, et al. Atezolizumab for first-line treatment of metastatic nonsquamous NSCLC. $N$ Engl $J$ Med. 2018;378(24):2288-2301. doi:10.1056/NEJMoa1716948

58. Cappuzzo F, McCleod M, Hussein M, et al. IMpower130: progression-free survival (PFS) and safety analysis from a randomised phase III study of carboplatin + nab-paclitaxel $(\mathrm{CnP})$ with or without atezolizumab (atezo) as first-line (1L) therapy in advanced non-squamous NSCLC. Ann Oncol. 2018;29(suppl_8): mdy424.065-mdy424.065. doi:10.1093/annonc/mdy424.065

59. Cherny NI, Dafni U, Bogaerts J, et al. ESMO-magnitude of clinical benefit scale version 1.1. Ann Oncol. 2017;28(10):2340-2366. doi:10.1093/annonc/mdx310

60. Waqar SN, Samson PP, Robinson CG, et al. Non-small-cell lung cancer with brain metastasis at presentation. Clin Lung Cancer. 2018;19(4):e373-e379. doi:10.1016/j.cllc.2018.01.007
61. Mansfield AS, Aubry MC, Moser JC, et al. Temporal and spatial discordance of programmed cell death-ligand 1 expression and lymphocyte tumor infiltration between paired primary lesions and brain metastases in lung cancer. Ann Oncol. 2016;27(10):1953-1958. doi:10.1093/annonc/mdw289

62. Mansfield AS, Ren H, Sutor S, et al. Contraction of T cell richness in lung cancer brain metastases. Sci Rep. 2018;8(1):2171. doi:10.1038/ s41598-018-20622-8

63. Hendriks L, Henon C, Mezquita L, et al. Impact of central nervous system (CNS) involvement in advanced non-small cell lung cancer (NSCLC) patients (pts) treated with immune checkpoint inhibitors (ICI). JCO. 2018;36(15_suppl):9066. doi:10.1200/ JCO.2018.36.15_suppl.9066

64. Crinò L, Bidoli $\bar{P}$, Ulivi P, et al. Italian Nivolumab Expanded Access Programme (EAP): data from patients with advanced non-squamous NSCLC and brain metastases. J Thorac Oncol. 2017;12(11):S1915. doi:10.1016/j.jtho.2017.09.707

65. Molinier O, Audigier-Valette C, Cadranel J, et al. IFCT-1502 CLINIVO: real-life experience with nivolumab in 600 patients (Pts) with Advanced Non-Small Cell Lung Cancer (NSCLC). J Thorac Oncol. 2017;12(11):S1793. doi:10.1016/j.jtho.2017.09.430

66. Gadgeel SM, Lukas RV, Goldschmidt J, et al. Atezolizumab in patients with advanced non-small cell lung cancer and history of asymptomatic, treated brain metastases: exploratory analyses of the phase III OAK study. Lung Cancer. 2019;128:105-112. doi:10.1016/j.lungcan.2018.12.017

67. Goldberg SB, Gettinger SN, Mahajan A, et al. Pembrolizumab for patients with melanoma or non-small-cell lung cancer and untreated brain metastases: early analysis of a non-randomised, open-label, phase 2 trial. Lancet Oncol. 2016;17(7):976-983. doi:10.1016/S1470-2045(16)30053-5

68. Tawbi HA, Forsyth PA, Algazi A, et al. Combined nivolumab and ipilimumab in melanoma metastatic to the Brain. $N$ Engl J Med. 2018;379(8):722-730. doi:10.1056/NEJMoa1805453

69. Long GV, Atkinson V, Lo S, et al. Combination nivolumab and ipilimumab or nivolumab alone in melanoma brain metastases: a multicentre randomised phase 2 study. Lancet Oncol. 2018;19 (5):672-681. doi:10.1016/S1470-2045(18)30139-6

70. Shaverdian N, Lisberg AE, Bornazyan K, et al. Previous radiotherapy and the clinical activity and toxicity of pembrolizumab in the treatment of non-small-cell lung cancer: a secondary analysis of the KEYNOTE-001 phase 1 trial. Lancet Oncol. 2017;18(7):895-903. doi:10.1016/S1470-2045(17)30380-7

71. Dovedi SJ, Adlard AL, Lipowska-Bhalla G, et al. Acquired resistance to fractionated radiotherapy can be overcome by concurrent PD-L1 blockade. Cancer Res. 2014;74(19):5458-5468. doi:10.1158/0008-5472. CAN-14-1258

72. Chen L, Douglass J, Kleinberg L, et al. Concurrent immune checkpoint inhibitors and stereotactic radiosurgery for brain metastases in non-small cell lung cancer, melanoma, and renal cell carcinoma. Int J Radiat Oncol Biol Phys. 2018;100(4):916-925. doi:10.1016/j.ijrobp.2017.11.041

73. Schapira E, Hubbeling H, Yeap BY, et al. Improved overall survival and locoregional disease control with concurrent PD-1 pathway inhibitors and stereotactic radiosurgery for lung cancer patients with brain metastases. Int $J$ Radiat Oncol Biol Phys. 2018;101 (3):624-629. doi:10.1016/j.ijrobp.2018.02.175

74. Hubbeling HG, Schapira EF, Horick NK, et al. Safety of combined PD-1 pathway inhibition and intracranial radiation therapy in non-small cell lung cancer. J Thorac Oncol. 2018;13(4):550-558. doi:10.1016/j.jtho.2018.01.012

75. Martin AM, Cagney DN, Catalano PJ, et al. Immunotherapy and symptomatic radiation necrosis in patients with brain metastases treated with stereotactic radiation. JAMA Oncol. 2018;4 (8):1123-1124. doi:10.1001/jamaoncol.2017.3993

76. Tallet AV, Dhermain F, Le Rhun E, Noël G, Kirova YM. Combined irradiation and targeted therapy or immune checkpoint blockade in brain metastases: toxicities and efficacy. Ann Oncol. 2017;28 (12):2962-2976. doi:10.1093/annonc/mdx408 
77. Ramalingam SS, Hellmann MD, Awad MM, et al. Tumor mutational burden (TMB) as a biomarker for clinical benefit from dual immune checkpoint blockade with nivolumab (nivo) + ipilimumab (ipi) in first-line (1L) non-small cell lung cancer (NSCLC): identification of TMB cutoff from CheckMate 568. Cancer Res. 2018;78(13 Supplement):CT078. doi:10.1158/1538-7445.AM2018-CT078

78. Muchnik E, Loh KP, Strawderman M, et al. Immune checkpoint inhibitors in real-world treatment of older adults with non-small cell lung cancer. J Am Geriatr Soc. 2019. doi:10.1111/jgs.15750

79. Champiat S, Dercle L, Ammari S, et al. Hyperprogressive disease is a new pattern of progression in cancer patients treated by anti-PD-1/ PD-L1. Clin Cancer Res. 2017;23(8):1920-1928. doi:10.1158/10780432.CCR-16-1741

80. Ferrara R, Mezquita L, Texier M, et al. Hyperprogressive disease in patients with advanced non-small cell lung cancer treated with PD-1/ PD-L1 inhibitors or with single-agent chemotherapy. JAMA Oncol. 2018;4(11):1543-1552. doi:10.1001/jamaoncol.2018.3676

81. Ferrara R, Naigeon M, Auclin E, et al. Immunosenescence (iSenescence) correlates with disease progression in advanced non-small cell lung cancer (aNSCLC) patients treated with PD-(L)1 inhibitors (IO). Ann Oncol. 2018;29(suppl_8):mdy292.038-mdy 292.038. doi:10.1093/annonc/mdy292.038

82. Reck M, Rodríguez-Abreu D, Robinson AG, et al. Updated analysis of KEYNOTE-024: pembrolizumab versus platinum-based chemotherapy for advanced non-small-cell lung cancer with PD-L1 tumor proportion score of $50 \%$ or greater. J Clin Oncol. 2019: JCO1800149. 10.1200/JCO.18.00149.

83. von Pawel J, Bordoni R, Satouchi M, et al. Long-term survival in patients with advanced non-small-cell lung cancer treated with atezolizumab versus docetaxel: results from the randomised phase III OAK study. Eur J Cancer. 2019;107:124-132. doi:10.1016/j.ejca.2018.11.020

84. Gettinger S, Horn L, Jackman D, et al. Five-year follow-up of nivolumab in previously treated advanced non-small-cell lung cancer: results from the CA209-003 study. J Clin Oncol. 2018;36 (17):1675-1684. doi:10.1200/JCO.2017.77.0412

85. Ricciuti B, Genova C, De Giglio A, et al. Impact of immune-related adverse events on survival in patients with advanced non-small cell lung cancer treated with nivolumab. J Thorac Oncol. 2018;13(10): S390-S391. doi:10.1016/j.jtho.2018.08.399

86. Moor R, Roberts KE, Mason R, et al. Immune-related adverse events and nivolumab outcome in non-small cell lung cancer patients: a multi-instutional, retrospective cohort study. J Clin Oncol. 2018;36 (suppl; abstr 9067).
87. Toi Y, Sugawara S, Kawashima Y, et al. Association of immune-related adverse events with clinical benefit in patients with advanced non-small-cell lung cancer treated with nivolumab. Oncologist. 2018. doi:10.1634/theoncologist.2017-0384

88. Haratani K, Hayashi H, Chiba Y, et al. Association of immune-related adverse events with nivolumab efficacy in non-small-cell lung cancer. JAMA Oncol. 2018;4(3):374-378. doi:10.1001/jamaoncol.2017.2925

89. Teraoka S, Fujimoto D, Morimoto T, et al. Early immune-related adverse events and association with outcome in advanced non-small cell lung cancer patients treated with nivolumab: a prospective cohort study. J Thorac Oncol. 2017;12(12):1798-1805. doi:10.1016/j. jtho.2017.08.022

90. Sato K, Akamatsu H, Murakami E, et al. Correlation between immune-related adverse events and efficacy in non-small cell lung cancer treated with nivolumab. Lung Cancer. 2018;115:71-74. doi:10.1016/j.lungcan.2017.11.019

91. Lisberg A, Tucker DA, Goldman JW, et al. Treatment-related adverse events predict improved clinical outcome in NSCLC patients on KEYNOTE-001 at a Single Center. Cancer Immunol Res. 2018. doi:10.1158/2326-6066.CIR-17-0063

92. von Pawel J, Syrigos K, Mazieres J, et al. Association between immune-related adverse events (irAEs) and atezolizumab efficacy in advanced NSCLC: analyses from the phase III study OAK. Ann Oncol. 2017;28(suppl_5):mdx380.017-mdx380.017. doi:10.1093/ annonc/mdx380.017

93. Kfoury M, Voisin AL, Nejean M, et al. Association between immune related adverse events and efficacy in patients treatd with anti-PD(L) 1. Ann Oncol. 2018;29(8):myd288.014. https://doi.org/10.1093/ annonc/mdy288.014

94. Toi Y, Sugawara S, Sugisaka J, et al. Profiling preexisting antibodies in patients treated with anti-PD-1 therapy for advanced non-small cell lung cancer. JAMA Oncol. 2018. doi:10.1001/jamaoncol.2018.5860

95. Spigel DR, McLeod M, Hussein MA, et al. Randomized results of fixed-duration (1-yr) vs continuous nivolumab in patients (pts) with advanced non-small cell lung cancer (NSCLC). Ann Oncol. 2017;28(suppl_5):mdx380.002-mdx380.002. doi:10.1093/annonc/ $\mathrm{mdx} 380.002$

\section{Publish your work in this journal}

Cancer Management and Research is an international, peer-reviewed open access journal focusing on cancer research and the optimal use of preventative and integrated treatment interventions to achieve improved outcomes, enhanced survival and quality of life for the cancer patient.
The manuscript management system is completely online and includes a very quick and fair peer-review system, which is all easy to use. Visit http://www.dovepress.com/testimonials.php to read real quotes from published authors. 